\title{
A note on nonparametric estimation of the effective dose in quantal bioassay
}

\author{
Holger Dette, Natalie Neumeyer, Kay F. Pilz \\ Ruhr-Universität Bochum \\ Fakultät für Mathematik \\ 44780 Bochum, Germany \\ e-mail: holger.dette@ruhr-uni-bochum.de
}

December 19, 2003

\begin{abstract}
For the common binary response model we propose a direct method for the nonparametric estimation of the effective dose level $\operatorname{ED}_{\alpha}(0<\alpha<1)$. The estimator is obtained by the composition of a nonparametric estimate of the quantile response curve and a classical density estimate. The new method yields a simple and reliable monotone estimate of the effective dose level curve $\alpha \rightarrow \mathrm{ED}_{\alpha}$ and is appealing to users of conventional smoothing methods as kernel estimators, local polynomials, series estimators or smoothing splines. Moreover it is computationally very efficient, because it does not require a numerical inversion of a monotonized estimate of the quantile dose response curve. We prove asymptotic normality of the new estimate and compare it with an available alternative estimate (based on a monotonized nonparametric estimate of the dose response curve and the calculation of the inverse function) by means of a simulation study.
\end{abstract}

AMS Subject Classification: 62G05, 62G20, 62P10

Keywords and Phrases: Binary response model, effective dose level, nonparametric regression, isotonic regression, order restricted inference, local linear regression

\section{Introduction}

Dose-response experiments are routinely conducted in preclinical and Phase I and II clinical trials to study the relationship between the dose level of a drug and the probability of a response, be it "cured" or "poisoned". The underlying assumption for quantal bioassay is that a subject is administered a stimulus at a dose level, say $x$, and that the response $Y$ is a binary random variable with success probability $p(x)$, i.e. $Y \sim \operatorname{Bin}(1, p(x))$. The function $p: \mathbb{R} \rightarrow[0,1]$ is called the dose 
response curve and is usually assumed to be strictly monotone. For a given $\alpha \in(p(0), p(1))$ the parameter of interest is the effective dose level

$$
\mathrm{ED}_{\alpha}=p^{-1}(\alpha)
$$

which is the dose, where $100 \alpha \%$ of the subjects react. If $n$ subjects are investigated at dose levels, say $x_{1}, \ldots, x_{n}$, the statistical model is given by

$$
P\left(Y_{i}=1 \mid x_{i}\right)=p\left(x_{i}\right)=1-P\left(Y_{i}=0 \mid x_{i}\right), \quad i=1, \ldots, n
$$

where different observations are assumed to be independent and the aim of the experiment is to estimate the effective dose level $\mathrm{ED}_{\alpha}$ for a particular $\alpha \in(p(0), p(1))$.

There are two basic approaches for this purpose, a parametric and nonparametric method. The first approach assumes a parametric form for the dose response curve, say $p(x)=p(x, \theta)$ for some $\theta \in \mathbb{R}^{k}$, and the unknown parameters are usually estimated by the maximum likelihood method. An estimate of the effective dose level is finally obtained by evaluating the inverse function $p^{-1}(\alpha, \hat{\theta})$. The most widely used models are the probit and the logit model for $p$ [see Berkson (1944) and Bliss (1934)], other models are the double exponential, the double reciprocal and the skewed logit model [see e.g. McCullagh and Nelder (1983)]. However, in many cases a parametric form of the dose response curve is not known by the experimenter, because the biological mechanisms are too complicated. In such cases the misspecification of a parametric model may lead to serious errors in the subsequent data analysis. For this reason numerous authors propose to estimate the effective dose level nonparametrically. Early proposals mainly for the purpose of estimating the $\mathrm{ED}_{0.5}$ nonparametrically can be found in Spearman (1908) and Thompson (1947). More recently Schmoyer (1984) constructed a constrained maximum likelihood estimator under the assumption that the dose response curve $p$ is sigmoid, while Müller and Schmitt (1988) proposed a kernel estimator for the dose response curve $p$. Because this estimate is not necessarily strictly monotone, it was first monotonized [see Barlow, Bartholomew, Bremner and Brunk (1972)] and the estimate of the effective dose level was then obtained by inversion according to (1.1).

In this note we discuss a more direct approach for the estimation of the effective dose levels. Our work is motivated by the search of a monotone estimate of the $\mathrm{ED}_{\alpha}$, which is not based on a constrained optimization and the inversion of the estimated dose response curve. For this purpose we construct a density estimate from the estimated dose response curve and use this additional smoothing step to obtain a monotone estimate of the effective dose level function directly.

The estimate is carefully described in Section 2, where we also discuss its main properties. In particular we prove asymptotic normality of the estimate for $p^{-1}$ and show that the new estimator is asymptotically first order equivalent to the estimate proposed by Müller and Schmitt (1988). Moreover, the calculation of the new estimate is substantially simpler than the currently available methods. In Section 3 we study some of the finite sample properties of our estimator by means of a simulation study and demonstrate that our new estimate is at least comparable to the currently available nonparametric estimates of the effective dose level. All technical details are deferred to an appendix.

We finally note that our approach has several advantages compared to the indirect methods proposed in the literature. The new procedure is based on a simple combination of a density and regression estimate. It is computational efficient and attractive to users of conventional kernel methods, 
because it does not require any constrained optimization techniques or inversion. Moreover, the indirect methods do not necessarily allow the estimation of the effective dose level $\mathrm{ED}_{\alpha}$ for any $\alpha \in(p(0), p(1))$, because it is not guaranteed that the inverse of the estimated dose response curve is defined for all $\alpha \in(p(0), p(1))$. Finally, the new estimator has an asymptotic normal distribution, which allows a very simple construction of asymptotic confidence intervals for the $\mathrm{ED}_{\alpha}$, whenever $\alpha \in(p(0), p(1))$.

\section{Monotone quantile estimation}

Consider the binary response model (1.2), where different observations are assumed to be independent and the dose response curve $p$ is assumed to be strictly increasing and two times continuously differentiable. For the experimental design $x_{1}<\ldots<x_{n}$ we assume without loss of generality

$$
\frac{\ell}{n}=\int_{0}^{x_{\ell}} f(t) d t, \quad \ell=1, \ldots, n
$$

for a positive two times continuously differentiable design density $f$ [see Sacks and Ylvisaker (1970)]. The nonparametric estimate of the effective dose level curve is defined by

$$
\hat{p}_{I}^{-1}(\alpha):=\frac{1}{N h_{d}} \sum_{i=1}^{N} \int_{-\infty}^{\alpha} K_{d}\left(\frac{\hat{p}\left(\frac{i}{N}\right)-u}{h_{d}}\right) d u, \quad \alpha \in(0,1)
$$

where $\hat{p}\left(\frac{i}{N}\right)$ is the local linear estimator at the point $\frac{i}{N}(i=1, \ldots, N)$, i.e. the component $\hat{\beta}_{i 0}$ of the vector $\left(\hat{\beta}_{i 0}, \hat{\beta}_{i 1}\right)$ which minimizes

$$
\sum_{k=1}^{n}\left\{Y_{k}-\beta_{i 0}-\beta_{i 1}\left(x_{k}-\frac{i}{N}\right)\right\}^{2} K_{r}\left(\frac{x_{k}-\frac{i}{N}}{h_{r}}\right)
$$

[see Wand and Jones (1995) or Fan and Gijbels (1996)]. In (2.2) and (2.3) $K_{d}$ and $K_{r}$ denote symmetric kernels with compact support, say $[-1,1]$, existing second moment and $h_{d}, h_{r}$ are the corresponding bandwidths converging to 0 with increasing sample size $n$. We also assume that $K_{d}$ is two times continuously differentiable and positive. The indices " $r$ " and " $d$ " correspond to the phrase "regression" and "density" because we combine a regression with a density estimate to define the estimator in (2.2). For the sake of transparency we restrict ourselves to the local linear estimate, but it is notable that all results of the paper remain valid (subject to an appropriate modification of constants) for other types of kernel estimators as the Gasser-Müller estimator [see Gasser and Müller (1979)] or higher order local polynomial estimators [see Fan and Gijbels (1996) or Wand and Jones (1995)]. It is also important to note that it is not necessary (and in many cases not desirable) that the number $N$ used in the density step coincides with the sample size $n$. The summation over the index $i$ can be considered as simple quadrature formula for the integral

$$
\frac{1}{h_{d}} \int_{0}^{1} \int_{-\infty}^{\alpha} K_{d}\left(\frac{\hat{p}(x)-u}{h_{d}}\right) d u d x
$$

and in cases, where this integral can be evaluated directly, a summation is in fact not necessary. 
Because the kernel $K_{d}$ is positive by assumption the estimate $\hat{p}_{I}^{-1}$ is obviously isotonic. Moreover, it is easy to see that the estimator $\hat{p}_{I}^{-1}$ is equal to 1 and 0 if $\alpha>\max _{i=1}^{N} \hat{p}\left(\frac{i}{N}\right)+h_{d}$ and $\alpha<$ $\min _{i=1}^{N} \hat{p}\left(\frac{i}{N}\right)-h_{d}$, respectively. Because $\hat{p}$ converges uniformly to the unknown dose response function $p$, it is heuristically clear that for $h_{d} \rightarrow 0$ the estimate $\hat{p}_{I}^{-1}$ is in some sense close to the function

$$
\frac{1}{h_{d}} \int_{0}^{1} \int_{-\infty}^{\alpha} K_{d}\left(\frac{p(x)-u}{h_{d}}\right) d u d x=\int_{0}^{1} I\{p(x) \leq \alpha\} d x+o(1)
$$

(note that the kernel $K_{d}$ has compact support). In other words, the statistic $\hat{p}_{I}^{-1}(\alpha)$ is a consistent and smooth estimate of the effective dose level $\mathrm{ED}_{\alpha}$

$$
\mathrm{ED}_{\alpha}=p^{-1}(\alpha)=\int_{0}^{1} I\{p(x) \leq \alpha\} d x,
$$

where the second equality follows from the strict monotonicity of the dose response curve $p$. If smoothing is not the main concern of the experimenter, the estimate

$$
\int_{0}^{1} I\{\hat{p}(x) \leq \alpha\} d x, \quad \alpha \in(0,1)
$$

or an approximation by a quadrature formula could be used alternatively. Note that this estimate is continous (if $\hat{p}$ is smooth), but not necessarily differentiable.

In the following discussion we make these heuristical arguments more precise. For this purpose we require the following assumptions regarding the bandwidths $h_{r}$ and $h_{d}$ in the regression and density estimate if the sample size $n$ tends to infinity:

$$
\begin{aligned}
& h_{r} \rightarrow 0, \quad h_{d} \rightarrow 0, \\
& n h_{d} \rightarrow \infty, \quad n h_{r} \rightarrow \infty \\
& \quad \lim _{h_{d} \rightarrow 0, h_{r} \rightarrow 0} h_{r} / h_{d}=\infty \\
& n h_{r}^{5}=O(1), \quad n=O(N), \\
& \frac{1}{n h_{r} h_{d}^{2}}=o(1)
\end{aligned}
$$

Note that in the case where the optimal bandwidth $h_{r}=\gamma n^{-1 / 5}$ is used for the estimation of the regression function condition (2.9) gives $h_{d} n^{2 / 5} \rightarrow \infty$. The following Lemma gives the asymptotic bias and variance of the estimate $\hat{p}_{I}^{-1}$.

Lemma 2.1. If the dose response curve is strictly increasing, conditions (2.5) - (2.9) and the assumptions stated at the beginning of this section are satisfied, then we have for any $\alpha \in(p(0), p(1))$ with $p^{\prime}\left(p^{-1}(\alpha)\right)>0$

$$
\begin{aligned}
E\left[\hat{p}_{I}^{-1}(\alpha)\right] & =b_{h_{d}, h_{r}}+o\left(h_{d}^{2}\right)+o\left(h_{r}^{2}\right)+O\left(\frac{1}{N h_{r}}\right), \\
\operatorname{Var}\left[\hat{p}_{I}^{-1}(\alpha)\right] & =\frac{g^{2}(\alpha)}{n h_{r}}+o\left(\frac{1}{n h_{r}}\right),
\end{aligned}
$$


where

$$
\begin{aligned}
b_{h_{d}, h_{r}} & =p^{-1}(\alpha)+\kappa_{2}\left(K_{d}\right) h_{d}^{2}\left(p^{-1}\right)^{\prime \prime}(\alpha)-\kappa_{2}\left(K_{r}\right) h_{r}^{2}\left(\frac{p^{\prime \prime}}{p^{\prime}}\right)\left(p^{-1}(\alpha)\right) \\
g^{2}(\alpha) & =\frac{\alpha(1-\alpha)}{\left\{p^{\prime}\left(p^{-1}(\alpha)\right)\right\}^{2} f\left(p^{-1}(\alpha)\right)} \int_{-1}^{1} K_{r}^{2}(u) d u
\end{aligned}
$$

and the constant $\kappa_{2}(K)$ is given by

$$
\kappa_{2}(K)=\frac{1}{2} \int_{-1}^{1} v^{2} K(v) d v .
$$

Note that it follows from assumption (2.7) that the bias of the estimate $\hat{p}_{I}^{-1}$ is asymptotically given by

$$
E\left[\hat{p}_{I}^{-1}(\alpha)-p^{-1}(\alpha)\right]=-\kappa_{2}\left(K_{r}\right) h_{r}^{2}\left(\frac{p^{\prime \prime}}{p^{\prime}}\right)\left(p^{-1}(\alpha)\right)+o\left(h_{r}^{2}\right)+O\left(\frac{1}{N h_{r}}\right)
$$

and that the asymptotic variance of $\hat{p}_{I}^{-1}$ has a similar structure as the variance of the common local linear estimator of a regression function [see e.g. Wand and Jones (1995)]. Our next theorem shows that the new estimator is asymptotically normal distributed.

Theorem 2.2. If the dose response curve is strictly increasing, conditions (2.5) - (2.9) and the assumptions stated at the beginning of this section are satisfied, then we have for any $\alpha \in(p(0), p(1))$ with $p^{\prime}\left(p^{-1}(\alpha)\right)>0$

$$
\sqrt{n h_{r}}\left(\hat{p}_{I}^{-1}(\alpha)-b_{h_{d}, h_{r}}\right) \stackrel{\mathcal{D}}{\Rightarrow} \mathcal{N}\left(0, g^{2}(\alpha)\right)
$$

where the asymptotic bias $b_{h_{d}, h_{r}}$ and variance $g^{2}(\alpha)$ are defined by (2.12) and (2.13), respectively.

Remark 2.3. Higher order asymptotic expansions for the bias can be obtained exactly in the same way. To be precise assume that $K_{d}$ is a kernel of even order $s \geq 2$ [see Gasser, Müller and Mammitzsch (1985)], that a local polynomial estimator of order $s-1$ is used for the regression estimate $\hat{p}$ [see Wand and Jones (1995) or Fan and Gijbels (1996)], and assume that the dose response curve is $s$-times continuously differentiable, then a similar calculation as given in the proof of Lemma 2.1 shows

$$
E\left[\hat{p}_{I}^{-1}(\alpha)-p^{-1}(\alpha)\right]=h_{d}^{s} \kappa_{s}\left(K_{d}\right)\left(p^{-1}\right)^{(s)}(\alpha)-h_{r}^{s} \kappa_{s}\left(K_{r}^{*}\right)\left(\frac{p^{(s)}}{p^{\prime}}\right)\left(p^{-1}(\alpha)\right)+o\left(h_{d}^{s}+h_{r}^{s}\right)
$$

where $\kappa_{s}(K)=\int_{-1}^{1} u^{s} K(u) d u / s$ ! and $K_{r}^{*}$ is the asymptotically equivalent kernel corresponding to the local polynomial estimator $\hat{p}$ [see Wand and Jones (1995) or Fan and Gijbels (1996)]. Moreover, the statement of asymptotic normality remains valid for this type of estimator, where the kernel $K_{r}$ in the asymptotic variance $(2.13)$ has to be replaced by $K_{r}^{*}$.

Remark 2.4. Müller and Schmitt (1988) proposed an alternative approach for estimating the effective dose level at some point $\alpha$. These authors used a monotonized kernel estimate of the 
dose response curve [see Brunk (1955), or Barlow, Bartholomew, Bremner and Brunk (1972)]. The final estimation of the effective dose level $\mathrm{ED}_{\alpha}$ was obtained by a numerical inversion of the isotonized estimate. The approach used in this paper performs the steps of isotonization and inversion simultanuously and has therefore some computational advantages. On the other hand a comparison of our Theorem 2.2 with Theorem 4 in Müller and Schmitt (1988) shows that the first order asymptotic properties of both estimates are exactly the same.

Note also that $\left(p^{-1}\right)^{\prime \prime}(\alpha)=-\left(p^{\prime \prime} /\left(p^{\prime}\right)^{3}\right)\left(p^{-1}(\alpha)\right)$ and consequently both terms in (2.12) are of the same sign. Therefore the bias of the estimator $\hat{p}_{I}^{-1}$ is slightly larger (depending on the order of $h_{d}$ ) than the bias of the estimator of Müller and Schmitt (1988), which is given by $-\kappa_{2}\left(K_{r}\right) h_{r}^{2}\left(p^{\prime \prime} / p^{\prime}\right)\left(p^{-1}(\alpha)\right)$. In concrete applications we recommend to use a bandwidth $h_{d}=O\left(h_{r}^{\beta}\right)$ where $\beta \in[1,5]$ and this effect is usually neglibile [see also our simulations in Section 3].

Remark 2.5. Although the new estimate and the estimate of Müller and Schmitt (1988) are asymptotically equivalent, there are some differences with respect to the practical performance of both procedures. First, the isotonized estimate of the dose response curve introduced by the lastnamed authors is not necessarily strictly isotone, which provides some difficulties in its numerical inversion [for a possible solution see Müller and Schmitt (1988) or Section 3 of the present paper, where a different approach is presented]. Secondly, the estimate of Müller and Schmitt (1988) cannot be calculated for any $\alpha \in(p(0), p(1))$, because it is not guaranteed that the range of isotonized kernel estimate of the dose response curve covers the whole interval $(p(0), p(1))$. For large sample sizes this phenomenon is negligible [provided that $\alpha \in(p(0), p(1))$ ], however for small and moderate sample sizes some problems could appear if estimation of the extreme effective dose levels is of interest. The new estimate $\hat{p}_{I}^{-1}$ avoids this problem by its different construction and yields estimates for any $\alpha \in(p(0), p(1))$.

Remark 2.6. Note that the new approach also provides simple estimates for the derivative of the effective dose level curve. For example,

$$
\left(\hat{p}_{I}^{-1}\right)^{\prime}(\alpha)=\frac{1}{N h_{d}} \sum_{i=1}^{N} K_{d}\left(\frac{\hat{p}\left(\frac{i}{N}\right)-\alpha}{h_{d}}\right)
$$

is a consistent estimator of $\left(p^{-1}\right)^{\prime}(\alpha)=\left(p^{\prime}\left(p^{-1}(\alpha)\right)\right)^{-1}$. Consequently, an asymptotic $(1-\alpha)$ confidence interval for the effective dose level $\mathrm{ED}_{\alpha}$ is given by $\left[\mathrm{ED}_{\alpha}^{-}, \mathrm{ED}_{\alpha}^{+}\right]$where

$$
\mathrm{ED}_{\alpha}^{ \pm}=\hat{p}_{I}^{-1}(\alpha)-\hat{b}_{h_{d}, h_{r}} \pm\left(\hat{p}_{I}^{-1}\right)^{\prime}(\alpha) \frac{u_{1-\alpha / 2}}{\sqrt{n h_{r}}} \sqrt{\frac{\alpha(1-\alpha)}{f\left(\hat{p}_{I}^{-1}(\alpha)\right)} \int K_{r}^{2}(u) d u}
$$

$u_{1-\alpha / 2}$ denotes the $(1-\alpha / 2)$-quantile of the standard normal distribution and

$$
\hat{b}_{h_{d}, h_{r}}=\kappa_{2}\left(K_{r}\right) h_{r}^{2} \frac{\left(\hat{p}_{I}^{-1}\right)^{\prime \prime}(\alpha)}{\left\{\left(\hat{p}_{I}^{-1}\right)^{\prime}(\alpha)\right\}^{2}}+\kappa_{2}\left(K_{d}\right) h_{d}^{2}\left(\hat{p}_{I}^{-1}\right)^{\prime \prime}(\alpha)
$$

is an estimate of the asymptotic bias, which can be omitted, if $h_{r}=o\left(n^{-1 / 5}\right)$ (note that $h_{d}=o\left(h_{r}\right)$ by assumption). 


\section{$3 \quad$ Finite sample properties}

In this section we investigate the finite sample properties of the new estimator $\hat{p}_{I}^{-1}$ for the effective dose levels. For the regression estimate $\hat{p}$ we use a local linear estimator with Epanechnikov kernel based on a uniform design $(f \equiv 1)$, where the bandwidth $h_{r}$ is chosen as

$$
\hat{h}_{r}=\left(\frac{\hat{\sigma}^{2}}{n}\right)^{1 / 5}
$$

and $\hat{\sigma}^{2}$ denotes the estimator of Rice (1984) that is

$$
\hat{\sigma}^{2}=\frac{1}{2(n-1)} \sum_{i=1}^{n-1}\left(Y_{i+1}-Y_{i}\right)^{2} .
$$

Note that this statistic converges almost surely to the quantity

$$
\sigma^{2}=\int_{0}^{1} p(x)(1-p(x)) d x
$$

[see Müller and Schmitt (1988)], which appears in the asymptotically optimal global bandwidth

$$
h_{r}=\left(\frac{\int_{-1}^{1} K_{r}^{2}(u) d u \int_{0}^{1} p(x)(1-p(x)) d x}{4 n \kappa_{2}^{2}\left(K_{r}\right) \int_{0}^{1}\left(p^{\prime \prime}(x)\right)^{2} d x}\right)^{1 / 5}
$$

for the estimation of the dose response curve under the uniform design. For the bandwidth $h_{d}$ we consider two cases, namely $h_{d}=h_{r}^{\beta}$, where $\beta=1.2$ and $\beta=2.0$. For the sake of comparison we also investigate a version of the estimator introduced by Müller and Schmitt (1988). More precisely we used the PAVA [Pool-Adjacent-Violators Algorithm, see Barlow, Bartholomew, Bremner and Brunk (1972)] to obtain an isotone estimate for the dose response curve. This estimate was then smoothed by a local linear estimator with bandwidth $\hat{h}_{r}$ in order to obtain a strictly isotonic and smooth estimate [see Mammen (1991)]. The final estimate which will be denoted by $\tilde{p}_{I}^{-1}$ throughout this section is obtained by a numerical inversion of this function. We investigate the binary response model (1.2) with a uniform design $(f \equiv 1)$ and the link functions

$$
\begin{aligned}
& p(x)=\Phi\left(\frac{x-\mu}{\sigma}\right), \quad \mu=.5, \sigma=.5 \\
& p(x)=\Phi\left(\frac{x-\mu}{\sigma}\right), \quad \mu=.5, \sigma=.1 \\
& p(x)=1-e^{-x^{\gamma}}, \quad \gamma=.52876 \\
& p(x)=\eta \Phi\left(\frac{x-\mu_{1}}{\tau}\right)+(1-\eta) \Phi\left(\frac{x-\mu_{2}}{\tau}\right), \\
& \mu_{1}=0.4, \mu_{2}=1.0, \eta=.64946, \tau=.13546
\end{aligned}
$$

which have also been considered by Müller and Schmitt (1988). The sample size is $n=48$, while the local linear estimate was evaluated $N=101$ points. 1000 simulation runs were performed for 
each scenario. In Figure 1-4 we present plots of the simulated bias, variance and mean squared error (mse) if $\alpha$ varies in the interval

$$
E=[p(0), p(1)]
$$

This guarantees the existence of $p^{-1}(\alpha)$ theoretically and the PAVA estimator can be calculated in most cases. We observe that there is no clear ordering between the different competitors. A comparison of the estimators $\hat{p}_{I}^{-1}$ with $h_{d}=h_{r}^{1.2}$ and $h_{d}=h_{r}^{2.0}$ yields that a larger exponent usually increases the variance and decreases the bias [see also Remark 2.4]. The effect on the mse depends on the particular model under consideration, but the differences are not substantial. A comparison of the new estimators with the estimator obtained from an inversion of the PAVA estimate shows a similar picture. The differences are not substantial and the superiority of a particular estimator depends on the model under consideration. For example in the Probit model (3.3) the PAVA method yields the estimator with the smallest mse, but the situation is reversed for the Probit model (3.4), where the estimator $\hat{p}_{I}^{-1}$ with $h_{d}=h_{r}^{1.2}$ should be preferred. Similarly, in the Weibull model (3.5) the new estimator $\hat{p}_{I}^{-1}$ yields a smaller mse for a broad range of $\alpha$, while the situation is less clear in model (3.6), where different estimators have to be preferred in different regions for the effective dose level. We finally note that in most cases the estimator $\hat{p}_{I}^{-1}$ yields a smaller mean squared error at the boundary of the interval $E$.

In most cases considered in our study the estimators behave very similar, which confirms the asymptotic results presented in Section 2. In many cases the new estimates have a slightly smaller variance at the cost of a slightly larger bias. Usually the new estimates yield a smaller mse at the boundary of $E$ and in the other regions of $E$ the statistic $\hat{p}_{I}^{-1}$ is at least competitive to the estimate obtained by the PAVA method, smoothing and numerical inversion (in many cases the mse of $\hat{p}_{I}^{-1}$ is even smaller). Taking into account the simplicity of the new estimator $\hat{p}_{I}^{-1}$ proposed in this paper we recommend its application if the effective dose level curve has to be estimated nonparametrically.

Example 3.1. Lee (1974) used the logistic regression model to determine characteristics associated with remission of cancer patients. The explanatory variable was the labeling index (LI), which measures proliferative activity of cells after a patient received an injection of tritiated thymidine [see also Agresti (1990) for a table of the data]. This index varies between 8 and 38 and the binary response variable measures if a patient received remission. There were 27 patients in the study, and under the assumption of a logistic regression model the estimate of the $\mathrm{ED}_{50}$ is given by 26.05 [Agresti (1990)]. The LI was linearly transformed to the interval [0,1], where the different estimators of the $\mathrm{ED}_{50}$ were applied. Finally the inverse linear transformation was applied to these estimates in order to obatin the estimates of the $\mathrm{ED}_{50}$ in the original scale. The bandwidth $h_{r}$ for the initial local linear estimate was chosen according to the rule of thumb (3.1), that is $h_{r} \approx 0.3557$ and this bandwidth was also used for the smoothing of the PAVA estimate. Finally the bandwidth $h_{d}$ in the density step was chosen as $h_{d}=h_{r}^{2} \approx 0.1265$. In Figure 5 we present plots of the estimates for the effective dose level curve obtained from the logistic regression, the PAVA estimator and the new estimator $\hat{p}_{I}^{-1}$. The PAVA estimate and the new estimate $\hat{p}_{I}^{-1}$ of the $\mathrm{ED}_{50}$ give very similar results, that is 21.78 and by 21.57 , respectively. The nonparametric estimators of the $\mathrm{ED}_{\alpha}$ behave very similar, if $\alpha<50 \%$ or $\alpha>70 \%$, while there are more differences if $\alpha$ varies in the interval $[60 \%, 70 \%]$. Moreover, the parametric and nonparametric methods yield rather different results for 
the estimation of the $\mathrm{ED}_{\alpha}$ in general, which indicates that the fit of the logistic regression model might not be appopriate for this data.

\section{Appendix: proofs}

For the sake of a transparent notation we assume that the number of design points $N$ in the estimate $\hat{p}_{I}^{-1}$ equals the sample size $n$. The general case can be obtained exactly in the same way with an additional amount of notation using assumption (2.8).

\subsection{Proof of Lemma 2.1.}

We use the decomposition

$$
\hat{p}_{I}^{-1}(\alpha)=\frac{1}{n h_{d}} \int_{-\infty}^{\alpha} \sum_{i=1}^{n} K_{d}\left(\frac{\hat{p}\left(\frac{i}{n}\right)-u}{h_{d}}\right) d u=p_{n}^{-1}(\alpha)+\Delta_{n}(\alpha),
$$

where the (non-random) function $p_{n}^{-1}$ is defined by

$$
p_{n}^{-1}(\alpha)=\frac{1}{n h_{d}} \int_{-\infty}^{\alpha} \sum_{i=1}^{n} K_{d}\left(\frac{p\left(\frac{i}{n}\right)-u}{h_{d}}\right) d u
$$

and the remainder $\Delta_{n}$ is given by

$$
\Delta_{n}(\alpha)=\frac{1}{n h_{d}} \sum_{i=1}^{n} \int_{-\infty}^{\alpha}\left\{K_{d}\left(\frac{\hat{p}\left(\frac{i}{n}\right)-u}{h_{d}}\right)-K_{d}\left(\frac{p\left(\frac{i}{n}\right)-u}{h_{d}}\right)\right\} d u
$$

Recall the definition of the local linear estimate

$$
\hat{p}(x)=\frac{1}{n h_{r}} \sum_{j=1}^{n} w\left(x_{j}, x, h_{r}\right) K_{r}\left(\frac{x_{j}-x}{h_{r}}\right) Y_{j},
$$

where

$$
w\left(x_{j}, x, h_{r}\right)=\frac{s_{2}\left(x, h_{r}\right)-s_{1}\left(x, h_{r}\right)\left(x_{j}-x\right)}{s_{2}\left(x, h_{r}\right) s_{0}\left(x, h_{r}\right)-s_{1}^{2}\left(x, h_{r}\right)}
$$

and

$$
s_{l}\left(x, h_{r}\right)=\frac{1}{n h_{r}} \sum_{j=1}^{n} K_{r}\left(\frac{x_{j}-x}{h_{r}}\right)\left(x_{j}-x\right)^{l}=\left\{\begin{array}{ll}
f(x)+o(1) & \text { if } l=0 \\
2 \kappa_{2}\left(K_{r}\right) f^{\prime}(x) h_{r}^{2}+o(1) & \text { if } l=1 \\
2 \kappa_{2}\left(K_{r}\right) f(x) h_{r}^{2}+o(1) & \text { if } l=2
\end{array} .\right.
$$

For the term (A.3) it follows that

$$
\Delta_{n}(\alpha)=\Delta_{n}^{(1)}(\alpha)+\frac{1}{2} \Delta_{n}^{(2)}(\alpha),
$$


where

$$
\begin{aligned}
\Delta_{n}^{(1)}(\alpha) & =\frac{1}{n h_{d}^{2}} \sum_{i=1}^{n} \int_{-\infty}^{\alpha} K_{d}^{\prime}\left(\frac{p\left(\frac{i}{n}\right)-u}{h_{d}}\right)\left\{\hat{p}\left(\frac{i}{n}\right)-p\left(\frac{i}{n}\right)\right\} d u \\
& =\frac{-1}{n h_{d}} \sum_{i=1}^{n} K_{d}\left(\frac{p\left(\frac{i}{n}\right)-\alpha}{h_{d}}\right)\left\{\frac{1}{n h_{r}} \sum_{j=1}^{n} w\left(x_{j}, \frac{i}{n}, h_{r}\right) K_{r}\left(\frac{x_{j}-\frac{i}{n}}{h_{r}}\right) Y_{j}-p\left(\frac{i}{n}\right)\right\}
\end{aligned}
$$

and

$$
\Delta_{n}^{(2)}(\alpha)=\frac{1}{n h_{d}^{3}} \sum_{i=1}^{n} \int_{-\infty}^{\alpha} K_{d}^{\prime \prime}\left(\frac{\xi_{i}-u}{h_{d}}\right)\left\{\hat{p}\left(\frac{i}{n}\right)-p\left(\frac{i}{n}\right)\right\}^{2} d u
$$

with $\left|\xi_{i}-p\left(\frac{i}{n}\right)\right|<\left|\hat{p}\left(\frac{i}{n}\right)-p\left(\frac{i}{n}\right)\right| \quad(i=1, \ldots, n)$. A straightforward calculation shows that

$$
E\left|\Delta_{n}^{(2)}(\alpha)\right|=\frac{1}{h_{d}^{2}}\left|\int_{0}^{1} K_{d}^{\prime}\left(\frac{p(x)-\alpha}{h_{d}}\right) E[\hat{p}(x)-p(x)]^{2} d x\right| \cdot(1+o(1))=o_{p}\left(\frac{1}{\sqrt{n h_{r}}}\right)
$$

where we used the fact that the expectation in the integral is of order $O\left(\frac{1}{n h_{r}}+h_{r}^{4}\right)$. A combination of (A.1), (A.7) and (A.10) shows that the first assertion of Lemma 2.1 can be proved establishing

$$
\begin{aligned}
p_{n}^{-1}(\alpha) & =p^{-1}(\alpha)+\kappa_{2}\left(K_{d}\right) h_{d}^{2}\left(p^{-1}\right)^{\prime \prime}(\alpha)+o\left(h_{d}^{2}\right)+O\left(\frac{1}{n h_{d}}\right), \\
E\left[\Delta_{n}^{(1)}(\alpha)\right] & =-h_{r}^{2} \kappa_{2}\left(K_{r}\right)\left(\frac{p^{\prime \prime}}{p^{\prime}}\right)\left(p^{-1}(\alpha)\right)+o\left(h_{r}^{2}\right) .
\end{aligned}
$$

For a proof of (A.11) we note that

$$
p_{n}^{-1}(\alpha)=A\left(h_{d}\right)\left(1+O\left(\frac{1}{n h_{d}}\right)\right)
$$

where

$$
\begin{aligned}
A\left(h_{d}\right) & =\int_{0}^{1} \int_{-\infty}^{\alpha} K_{d}\left(\frac{p(x)-u}{h_{d}}\right) \frac{d u}{h_{d}} d x \\
& =p^{-1}\left(\alpha-h_{d}\right)+h_{d} \int_{\frac{p(0)-\alpha}{h_{d}}}^{\frac{p(1)-\alpha}{h_{d}}} I\{-1 \leq z \leq 1\}\left(p^{-1}\right)^{\prime}\left(\alpha+z h_{d}\right) \int_{z}^{1} K_{d}(v) d v d z .
\end{aligned}
$$

If $\alpha \in(p(0), p(1))$ is fixed, we obtain from the identity $\int_{-1}^{1} \int_{z}^{1} K_{d}(v) d v d z=1$ (note that $K_{d}$ is symmetric and has compact support $[-1,1])$ and a Taylor expansion

$$
\begin{aligned}
A\left(h_{d}\right) & =p^{-1}(\alpha)+h_{d}^{2}\left(p^{-1}\right)^{\prime \prime}(\alpha)\left\{\frac{1}{2}+\int_{-1}^{1} z \int_{z}^{1} K_{d}(v) d v d z\right\}+o\left(h_{d}^{2}\right) \\
& =p^{-1}(\alpha)+\kappa_{2}\left(K_{d}\right) h_{d}^{2}\left(p^{-1}\right)^{\prime \prime}(\alpha)+o\left(h_{d}^{2}\right)
\end{aligned}
$$


as $h_{d} \rightarrow 0$, where we used the representation $\int_{-1}^{1} z \int_{z}^{1} K_{d}(v) d v d z=\frac{1}{2} \int_{-1}^{1} v^{2} K_{d}(v) d v-\frac{1}{2}$. This proves (A.11). For a proof of representation (A.12) we introduce the decomposition

$$
\Delta_{n}^{(1)}(\alpha)=\left(\Delta_{n}^{(1.1)}(\alpha)+\Delta_{n}^{(1.2)}(\alpha)\right)
$$

where

$$
\begin{aligned}
& \Delta_{n}^{(1.1)}(\alpha)=\frac{-1}{n h_{d}} \sum_{i=1}^{n} K_{d}\left(\frac{p\left(\frac{i}{n}\right)-\alpha}{h_{d}}\right)\left\{\frac{1}{n h_{r}} \sum_{j=1}^{n} w\left(x_{j}, \frac{i}{n}, h_{r}\right) K_{r}\left(\frac{x_{j}-\frac{i}{n}}{h_{r}}\right) p\left(x_{j}\right)-p\left(\frac{i}{n}\right)\right\} \\
& \Delta_{n}^{(1.2)}(\alpha)=\frac{-1}{n^{2} h_{d} h_{r}} \sum_{i, j=1}^{n} K_{d}\left(\frac{p\left(\frac{i}{n}\right)-\alpha}{h_{d}}\right) K_{r}\left(\frac{x_{j}-\frac{i}{n}}{h_{r}}\right)\left(Y_{j}-p\left(x_{j}\right)\right) w\left(x_{j}, \frac{i}{n}, h_{r}\right) .
\end{aligned}
$$

The second term has obviously expectation 0 and therefore the expectation of $\Delta_{n}^{(1)}(\alpha)$ is given by

$$
\begin{aligned}
E\left[\Delta_{n}^{(1)}(\alpha)\right] & =E\left[\Delta_{n}^{(1.1)}(\alpha)\right] \\
& =-\frac{1}{n h_{d}} \sum_{i=1}^{n} K_{d}\left(\frac{p\left(\frac{i}{n}\right)-\alpha}{h_{d}}\right) \mathrm{E}\left[\hat{p}\left(\frac{i}{n}\right)-p\left(\frac{i}{n}\right)\right] \\
& =-h_{r}^{2} \kappa_{2}\left(K_{r}\right) \int_{0}^{1} \frac{1}{h_{d}} K_{d}\left(\frac{p(x)-\alpha}{h_{d}}\right) p^{\prime \prime}(x) d x \cdot(1+o(1)) \\
& =-h_{r}^{2} \kappa_{2}\left(K_{r}\right)\left(\frac{p^{\prime \prime}}{p^{\prime}}\right)\left(p^{-1}(\alpha)\right) \cdot(1+o(1))
\end{aligned}
$$

which proves (A.12) and the assertion of Lemma 2.1 regarding the expectation of the estimator $\hat{p}_{I}^{-1}$. For a proof of the corresponding statement for the variance we note that it follows from (A.1), (A.7) and similar arguments as used in the derivation of (A.10) that

$$
\operatorname{Var}\left(\hat{p}_{I}^{-1}(\alpha)\right)=\operatorname{Var}\left(\Delta_{n}^{(1)}(\alpha)\right)+o\left(\frac{1}{n h_{r}}\right)
$$

where $\Delta_{n}^{(1)}$ is defined in (A.8). The same arguments as given in the previous paragraph show for the random variable $\Delta_{n}^{(1.1)}(\alpha)$ defined in (A.15)

$$
\operatorname{Var}\left[\left(\Delta_{n}^{(1.1)}(\alpha)\right)^{2}\right]=o\left(\frac{1}{n h_{r}}\right)
$$

and we obtain from (A.16), (A.5) and (A.6)

$$
\begin{array}{r}
\operatorname{Var}\left[\Delta_{n}^{(1.2)}(\alpha)\right]=\frac{1}{n^{4} h_{d}^{2} h_{r}^{2}} \sum_{j=1}^{n} \operatorname{Var}\left(\sum_{i=1}^{n}\left(Y_{j}-p\left(x_{j}\right)\right) K_{d}\left(\frac{p\left(\frac{i}{n}\right)-\alpha}{h_{d}}\right) K_{r}\left(\frac{x_{j}-\frac{i}{n}}{h_{r}}\right) w\left(x_{j}, \frac{i}{n}, h_{r}\right)\right) \\
=\int_{0}^{1} \frac{p(x)(1-p(x))}{n h_{d}^{2} h_{r}^{2}}\left[\int_{0}^{1} K_{d}\left(\frac{p(y)-\alpha}{h_{d}}\right) K_{r}\left(\frac{x-y}{h_{r}}\right) w\left(x, y, h_{r}\right) d y\right]^{2} f(x) d x \\
\times(1+o(1))
\end{array}
$$




$$
\begin{aligned}
= & \frac{1}{n h_{d}^{2} h_{r}^{2}} \int_{0}^{1} K_{d}\left(\frac{p(z)-\alpha}{h_{d}}\right) \int_{0}^{1} K_{d}\left(\frac{p(y)-\alpha}{h_{d}}\right)\left\{\frac{1}{f(y)}-\frac{f^{\prime}(y)}{f^{2}(y)}(x-y)\right\} \\
& \times\left\{\frac{1}{f(z)}-\frac{f^{\prime}(z)}{f^{2}(z)}(x-z)\right\} \int_{0}^{1} p(x)(1-p(x)) K_{r}\left(\frac{x-y}{h_{r}}\right) K_{r}\left(\frac{x-z}{h_{r}}\right) f(x) d x d y d z \\
= & \frac{\alpha(1-\alpha)}{n h_{r} p^{\prime}\left(p^{-1}(\alpha)\right)^{2} f\left(p^{-1}(\alpha)\right)} \iiint K_{d}(w) K_{d}(v) K_{r}(u) \\
& \times K_{r}\left(\frac{p^{-1}\left(\alpha+h_{d} v\right)-p^{-1}\left(\alpha+h_{d} w\right)}{h_{r}}+u\right) d u d v d w \cdot(1+o(1)) \\
= & \frac{\alpha(1-\alpha)}{n h_{r}\left\{p^{\prime}\left(p^{-1}(\alpha)\right)\right\}^{2} f\left(p^{-1}(\alpha)\right)} \int K_{r}^{2}(u) d u \cdot(1+o(1)),
\end{aligned}
$$

where we used assumption (2.7) and the differentiability of $p^{-1}$ for the last identity. This yields

$$
\operatorname{Var}\left(\hat{p}_{I}^{-1}(\alpha)\right)=\operatorname{Var}\left(\Delta_{n}^{(1.2)}(\alpha)\right)(1+o(1))=\frac{\alpha(1-\alpha)}{n h_{r}\left(p^{\prime}\left(p^{-1}(\alpha)\right)\right)^{2} f\left(p^{-1}(\alpha)\right)} \int_{-1}^{1} K_{r}^{2}(u) d u
$$

which proves the representation of the asymptotic variance in Lemma 2.1.

\subsection{Proof of Theorem 3.1.}

Using the notation and the arguments from the proof of Lemma 2.1 if follows from (A.1), (A.7), (A.10), (A.11), (A.12), and (A.19) that

$$
\sqrt{n h_{r}}\left(\hat{p}_{I}^{-1}(\alpha)-b_{h_{d}, h_{r}}\right)=\sqrt{n h_{r}} \Delta_{n}^{(1.2)}(\alpha)+o_{p}(1)
$$

where $\Delta_{n}^{(1.2)}(\alpha)$ is a sum of independent random variables, which is defined in (A.16). The asymptotic normality can now easily be obtained from Ljapunoff's theorem. To this end we note that $\operatorname{Var}\left[\Delta_{n}^{(1.2)}(\alpha)\right]=O\left(\frac{1}{n h_{r}}\right)$ by Lemma 2.1 and calculate by similar arguments as given in the proof of Lemma 2.1

$$
\begin{aligned}
& \sum_{j=1}^{n} E\left[\left\{\frac{Y_{j}-p\left(x_{j}\right)}{n^{2} h_{d} h_{r}} \sum_{i=1}^{n} K_{d}\left(\frac{p\left(\frac{i}{n}\right)-\alpha}{h_{d}}\right) K_{r}\left(\frac{x_{j}-\frac{i}{n}}{h_{r}}\right) w\left(x_{j}, \frac{i}{n}, h_{r}\right)\right\}^{4}\right] \\
& =\frac{1}{n^{3} h_{d}^{4} h_{r}^{4}} \int \gamma(x)\left\{\prod_{j=1}^{4} \int K_{d}\left(\frac{p\left(x_{j}\right)-\alpha}{h_{d}}\right) K_{r}\left(\frac{x-x_{j}}{h_{r}}\right) w\left(x, x_{j}, h_{r}\right) d x_{j}\right\} d x \cdot(1+o(1)) \\
& =\frac{1}{n^{3} h_{r}^{4}} \int \gamma(x)\left\{\prod_{j=1}^{4} \int K_{d}\left(y_{j}\right) K_{r}\left(\frac{x-p^{-1}\left(\alpha+h_{d} y_{j}\right)}{h_{r}}\right) \frac{\left(p^{-1}\right)^{\prime}\left(\alpha+h_{d} y_{j}\right) d y_{j}}{f\left(\alpha+h_{d} y_{j}\right)}\right\} d x \\
& =O\left(\frac{1}{\left(n h_{r}\right)^{3}}\right),
\end{aligned}
$$


where $\gamma\left(x_{j}\right)=\mathrm{E}\left[\left(Y_{j}-p\left(x_{j}\right)\right)^{4}\right]$. The asymptotic normality now follows from (A.21) and the central limit theorem of Ljapunoff.

Acknowledgements. The authors are grateful to Isolde Gottschlich who typed parts of this paper with considerable technical expertise and to E. Mammen and W. Polonik for useful discussions and some help with the references. The work of the authors was supported by the Sonderforschungsbereich 475, Komplexitätsreduktion in multivariaten Datenstrukturen.

\section{References}

A. Agresti (1990) Categorical Data Analysis. Wiley, N.Y.

R.E. Barlow, D.J. Bartholomew, J.M. Bremner, H.D. Brunk (1972). Statistical Inference under Order Restrictions. John Wiley \& Sons, London.

J. Berkson (1944). Application of the Logistic Function to Bioassay. J. Amer. Statist. Assoc., 39, 357-365.

C.I. Bliss (1934). The Method of Probits. Science, 79, 38-39.

H.D. Brunk (1955). Maximum likelihood estimates of monotone parameters. Ann. Math. Statist. $26,607-616$.

J. Fan, I. Gijbels (1996). Local polynomial modelling and its applications. Chapman and Hall, London.

T. Gasser, H.G. Müller (1979). Kernel estimation of regression functions. Lect. Notes Math. 757, 23-68 .

T. Gasser, H.G. Müller, V. Mammitzsch (1985). Kernels for nonparametric curve estimation. J. Roy. Statist. Soc. Ser. B, 47, no. 2, 238-252.

E.T. Lee (1974). A computer program for linear logistic regression analysis. Computer Prog. Biomed. 4, 80-92

E. Mammen (1991). Estimating a smooth monotone regression function. Ann. Statist. 19, 724-740.

P. McCullagh, J.A. Nelder (1983). Generalized Linear Models. Chapman\&Hall, London.

H.G. Müller, T. Schmitt (1988). Kernel and probit estimates in quantal bioassay. J. Amer. Statist. Assoc. 83, 750-759.

J. Rice (1984). Bandwidth choice for nonparametric regression. Ann. Statist. 12, 1215-1230.

J. Sacks, D. Ylvisaker (1970). Designs for regression problems for correlated errors. Ann. Math. Statist., 41, 2057-2074.

R.L. Schmoyer (1984). Sigmoidally constrained maximum likelihood estimation in quantal bioassay. J. Amer. Statist. Assoc. 79, 448-453. 
C. Spearman (1908). The Method of Right and Wrong Cases (Constant Stimuli) Without Gauss's Formulae. British J. Psychology, 2, 227-242.

W.R. Thompson (1947). Use of Moving Averages and Interpolation to Estimate Median Effective Dose, I: Fundamental Formulas, Estimation of Error, and Relation to Other Methods. Bacteriological Reviews, 11, 116-145.

M.P. Wand, M.G. Jones (1995). Kernel smoothing. Chapman and Hall, London. 

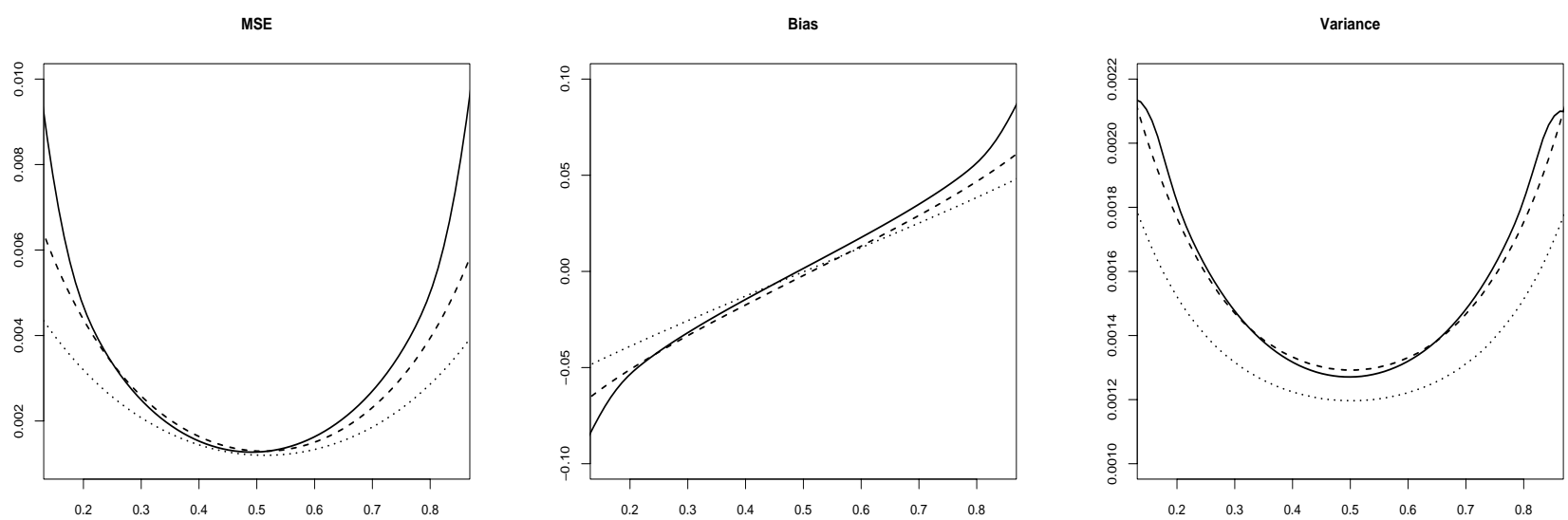

Figure 1: Probit model (3.3). Simulated mean squared error, bias and variance of the estimator $\hat{p}_{I}^{-1}$ with $h_{d}=h_{r}^{1.2}$ (solid line) and $h_{d}=h_{r}^{2.0}$ (dashed line) and of the estimator obtained from an inversion of a smooth PAVA estimate (dotted line).
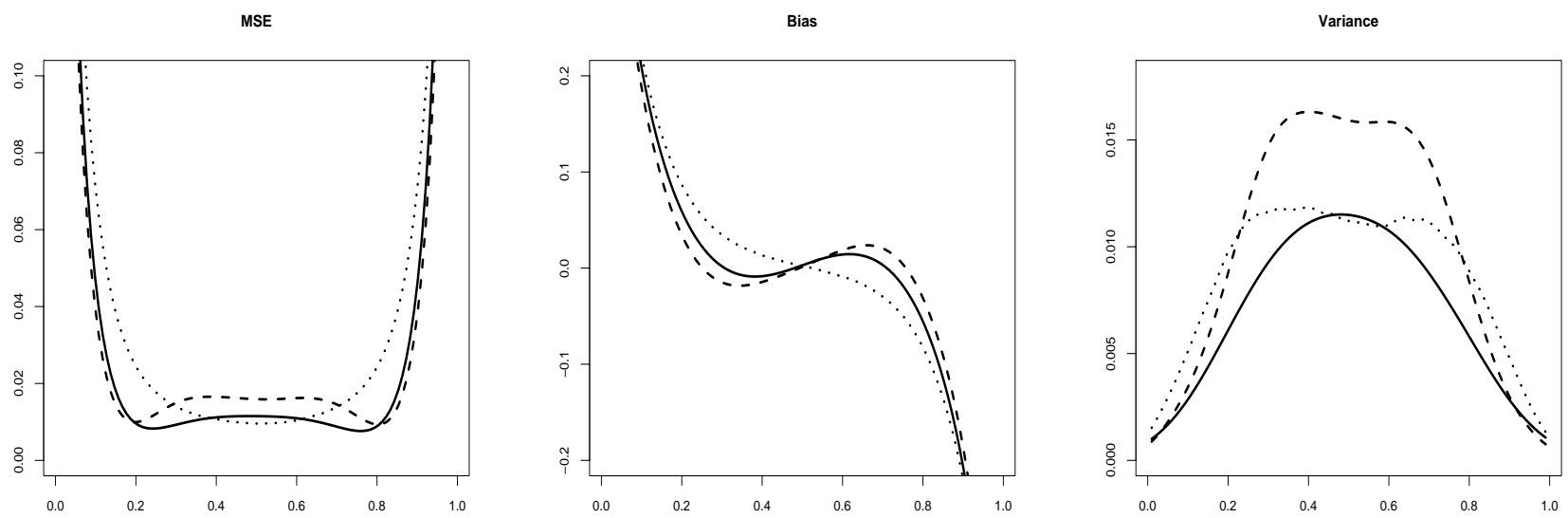

Figure 2: Probit model (3.4). Simulated mean squared error, bias and variance of the estimator $\hat{p}_{I}^{-1}$ with $h_{d}=h_{r}^{1.2}$ (solid line) and $h_{d}=h_{r}^{2.0}$ (dashed line) and of the estimator obtained from an inversion of a smooth PAVA estimate (dotted line). 

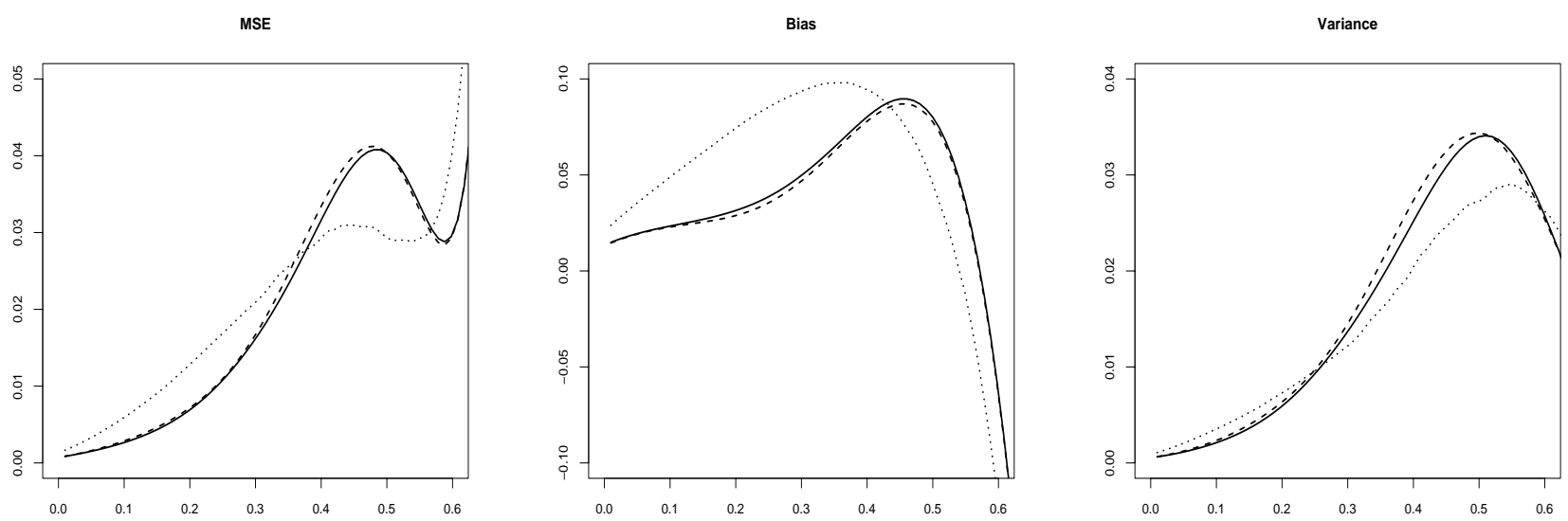

Figure 3: Weibull model (3.5). Simulated mean squared error, bias and variance of the estimator $\hat{p}_{I}^{-1}$ with $h_{d}=h_{r}^{1.2}$ (solid line) and $h_{d}=h_{r}^{2.0}$ (dashed line) and of the estimator obtained from an inversion of a smooth PAVA estimate (dotted line).
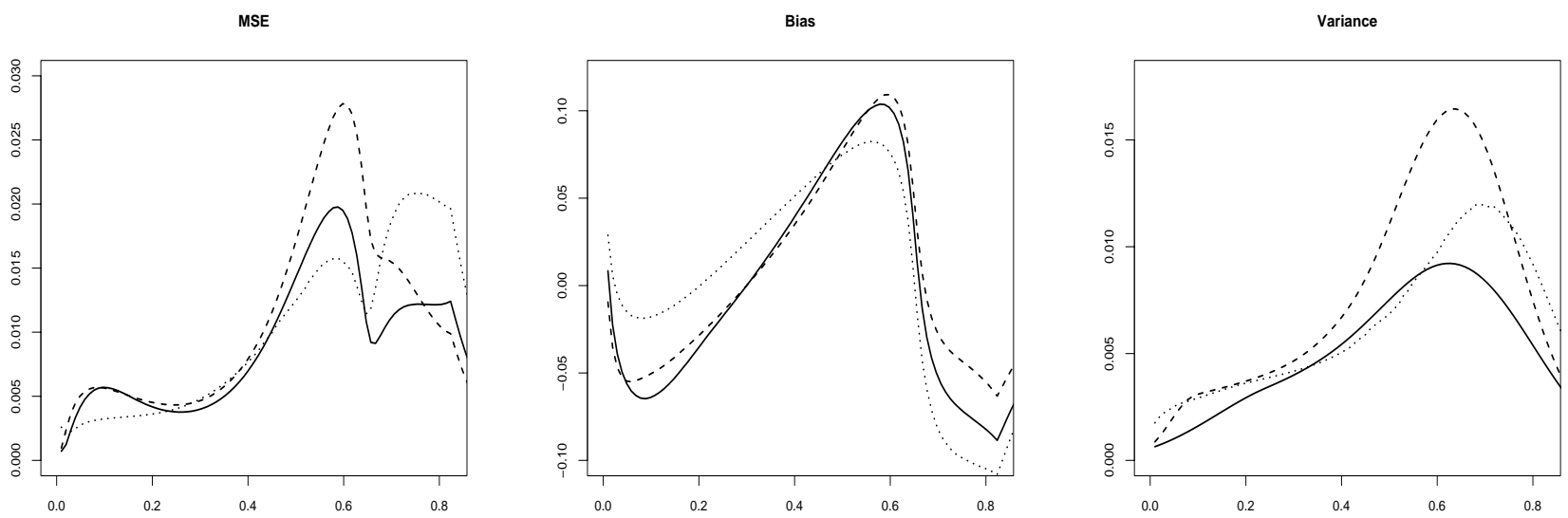

Figure 4: Normal mixture model (3.6). Simulated mean squared error, bias and variance of the estimator $\hat{p}_{I}^{-1}$ with $h_{d}=h_{r}^{1.2}$ (solid line) and $h_{d}=h_{r}^{2.0}$ (dashed line) and of the estimator obtained from an inversion of a smooth PAVA estimate (dotted line). 


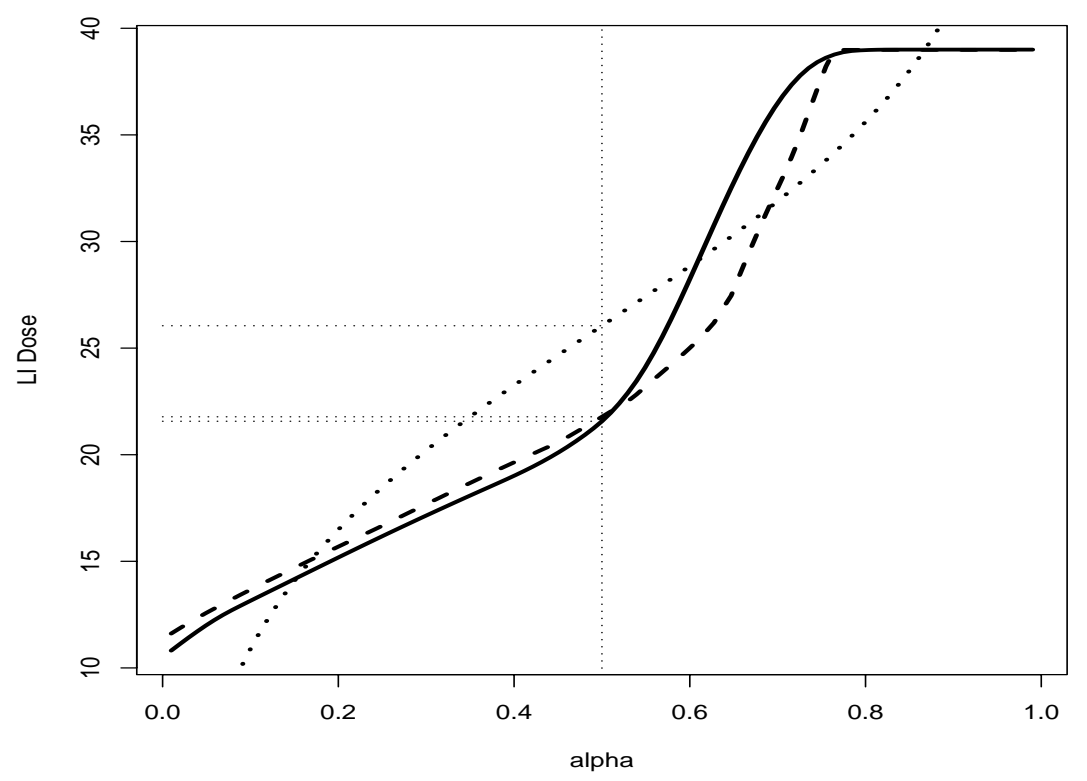

Figure 5: Estimated effective dose level curves in cancer remission Example 3.1. Dotted line: logistic regression; dashed line: inverse of the smoothed PAVA estimate $\tilde{p}_{I}^{-1}$; solid line: the new estimate $\hat{p}_{I}^{-1}$. The three estimates of the ED $D_{50}$ are indicated by the horizontal lines. 